\title{
Identification and validation of mutator-derived IncRNA signatures of genome instability for predicting prognosis of Pancreatic Cancer patients
}

\section{Aman Xu ( $\sim$ xuaman@ahmu.edu.cn )}

First Affiliated Hospital of Anhui Medical University

\section{Baichuan Zhou}

First Affiliated Hospital of Anhui Medical University

Xiaodong Zhu

First Affiliated Hospital of Anhui Medical University

Lixiang Zhang

First Affiliated Hospital of Anhui Medical University

\section{Panquan Luo}

First Affiliated Hospital of Anhui Medical University

\section{Zhijian Wei}

First Affiliated Hospital of Anhui Medical University

\section{Wenxiu Han}

First Affiliated Hospital of Anhui Medical University

\section{Research Article}

Keywords:

Posted Date: February 23rd, 2022

DOI: https://doi.org/10.21203/rs.3.rs-1369703/v1

License: (c) (i) This work is licensed under a Creative Commons Attribution 4.0 International License. Read Full License 


\section{Abstract}

Background: Currently, the long-term prognosis of pancreatic cancer (PC) is difficult to accurately predict. Therefore, the purpose of this study is to identify IncRNA associated with genome instability, and establish genome instability-derived IncRNA signature (GILncSig).

Method: Clinical information, RNA-seq expression data and somatic mutation data were acquired from The Cancer Genome Atlas (TCGA) database. The GO and KEEG analysis was applied to built IncRNAsmRNA co-expression network was associated with the genome instability.

Univariate and multivariate Cox proportional hazard regression were used to single out the independently prognostic IncRNA in PC patients. Finally, 9 Incrnas were discovered and incorporated into the construction of GILncSig. Patients were divided into high-risk and low-risk groups based on the median risk score, and Kaplan-Meier survival analysis was used to compare the overall survival between the two groups.

Results: KEEG analysis are mainly enriched in a variety of cell signal transduction and metabolism. The risk score of GILncSig was built by 9 IncRNA (SOCS2-AS1, AC127024.6, TRPC7-AS1, AL359504.1, AL117382.2, LYPLAL1-AS1, LINC02014, CASC8, KRT7-AS), and the Kaplan-Meier curve showed that the prognosis of low risk patient were better than high risk patients in the testing group $(p=0.003)$ and the whole TCGA patients $(p<0.001)$. According to the assessment of genome instability, KRAS wide-type patients was divided into two groups, with the genome stable group showing significantly improved outcome and the genome unstable group showing no significant difference compared with those with KRAS mutation.

Conclusion: This study provides a prognostic model for predicting the prognosis of PC patients, and introduces reference and evidence of the role of IncRNA in genome instability in the further study.

\section{Introduction}

Pancreatic cancer (PC) is one of the fatal malignancies in the world and have a high mortality with the 5-

year survival rate below $5 \%{ }^{[1,2]}$. Radical resection with lymph node cleaning is considered the only means of curative treatment of patients ${ }^{[3]}$, which provides a chance of longer survival. In recent years, multimodal treatments have been taken with the tumors, the prognosis of patients are still poor because of the hidden location and untypical clinical symptoms. CA19-9 is the only biomarker which play important role in early diagnosis, and assessment of therapeutic effect ${ }^{[4]}$, the sensitivity and specificity are not great. Therefore, it is necessary to further study the molecular mechanism and new therapeutic targets for PC.

Molecular elements, including miRNA, IncRNA and protein coding genes affected by copy number variation, which may predispose affected cells to oncogenesis ${ }^{[5]}$. Previous studies showed that the genome instability and variation can resulted in effects on the tumorigenesis of $\mathrm{PC}{ }^{[6]}$, which are related 
with the prognosis and disease progression ${ }^{[7]}$. For example, Wang et al ${ }^{[8]}$ constructed an miRNAregulatory network that is associate with genome instability and survival of ovarian cancer patients. The term IncRNAs are defined as transcripts with lengths that exceed 200 nucleotides, recent studies have verified IncRNA play significant role in biological process, including cell differentiation, proliferation and invasion ${ }^{[9]}$. With the rapid development of the next generation sequencing technology, the function of potential IncRNA has explored, some researches found IncRNA contributes to genome stability by interacting with DNA damage proteins or regulating the expression of DNA damage-related genes ${ }^{[10,11]}$. Comprehensive analyses of expression profiles of IncRNA with genome instability and clinical cancer outcomes, would identify IncRNAs associated with specific cancers. The knowledge of IncRNA with genome instability in PC is limited.

In this study, we utilized bioinformatics strategies to assess the relationship

between IncRNAs expression profiles and genome instability in PC patients, besides we identify significant IncRNAs to reflect the prognosis of PC, thereby improving its prognostic value.

\section{Materials And Methods}

\section{Data collection}

Clinical information, RNA-seq expression data and somatic mutation data were acquired from The Cancer Genome Atlas (TCGA) database (https://portal.gdc.cancer.gov/). GU(Genome Unstable)-like group and GS(Genome Stable)-like group were screened from 178 sample of TCGA, 353 IncRNA were significantly different in the two groups. And the patients with clinical information were grouped into training set and testing set by removing some patients because of the follow-up within 30 days or incomplete clinical data. Finally 9 IncRNA were found related with the survival of training set and an GILncSig was built by prognostic IncRNA, then this model was verified in the testing group and the whole TCGA patients.

\section{Statistical analysis}

Univariate and multivariate Cox proportional hazard regression were used to identify significant IncRNA in PC patients. The genome instability-derived IncRNA signature (GILncSig) was built and calculated by prognostic IncRNA as follows:

expr (IncRNAi) is the expression level of prognostic IncRNA and coef (IncRNAi) represents the contribution of IncRNA obtained from the regression coefficient of multivariate Cox analysis. The Kaplan-Meier method and log-rank test were used to compare the survival rate of different group, and the cox regression was used to explore the prognostic factor of survival, The performance of the GILncSig was expressed and compared with other model by receiver operating characteristic(ROC) curve. $P<0.05$ was significant, and the whole statistical analyses were performed using R-version 4.0.2.

\section{Results}




\section{Identification of genome instability-related IncRNAs in PC patients}

The cumulative number of somatic mutations for every sample was calculated, and the top $25 \%(n=45)$ and the last $25 \%(n=46)$ of the samples were grouped to GU-like group and GS-like group according to the mutation frequency. Then IncRNA was compared in the two groups, 353 IncRNA were significantly different with /fold change value/ $>1$ and FDR-adjusted P-value $<0.05$. The Detailed information of upregulated and downregulated IncRNA was expressed in the Table 1. And the top 20 of upregulated and downregulated IncRNA was showed in the Fig. 1. Hierarchical clustering analysis was conducted on 178 sample of PC according the different IncRNA(Fig. 2A), all samples were clustered as GU-like group and GS-like group, and the somatic mutation count in the two group were significant $(p<0.001$, Fig. $2 B)$, besides, the expression of UBQLN4 which can drive genome instability is higher in GU-like group than GSlike group $(p<0.001$, Fig. $2 C)$. The top 10 mRNA correlated with each different IncRNA were got and then we built an IncRNAs-mRNA co-expression network(Fig. 2D). The GO and KEEG analysis of related mRNA were showed as Fig. 2E indicating that IncRNAs-mRNA co-expression network was associated with the genome instability.

\section{The GILncSig built by prognostic IncRNA}

All samples of PC from the TCGA project were divided randomly into the training $(n=87)$ and testing group $(n=84)$. In the training group, univariate and multivariate cox proportional hazards regression analysis were conducted to single out the independently prognostic IncRNA, finally 9 IncRNA were found related with the survival of PC(Table 2, SOCS2-AS1, AC127024.6, TRPC7-AS1, AL359504.1, AL117382.2, LYPLAL1-AS1, LINC02014, CASC8, KRT7-AS). And the risk score of genome instability-derived IncRNA signature (GILncSig) was constructed and calculated as follows: (1.5965*SOCS2-AS1)+( $1.2868 * A C 127024.6)+(-0.8692 *$ TRPC7-AS1 $)+(-2.7202 * A L 359504.1)+(-0.9616 * A L 117382.2)+($ $0.1773 *$ LYPLAL1-AS1 $)+(0.8697 *$ LINC02014 $)+(0.2219 *$ CASC8 $)+(0.0862 *$ KRT7-AS $)$. Then the risk score of each patients was acquired, according to the median risk score of training group, the patients were divided into high risk group and low risk group. The Kaplan-Meier curve showed that the prognosis of high risk patient were poorer than low risk patients $(p<0.001)$ (Fig. 3A), The time-dependent ROC curves was drew and the area under curve (AUC) of GILncSig in the training group is 0.788 (Fig. 3B). The expression of 9 significant IncRNA, the count of somatic mutations and the level of UBQLN4 were observed with the change of risk score(Fig. 3C).

In order to verify the results of GILncSig in the training group, we also do the same methods in the testing group and the whole TCGA patients. According to the median risk score of each group, the testing group and the whole patients were divided into high risk group and low risk group, respectively. The KaplanMeier curve showed that the prognosis of low risk patient were better than high risk patients in the testing group $(p=0.003)$ and the whole TCGA patients $(p<0.001)(F i g .4 A, C)$. The AUC of time-dependent ROC curves in the testing group and the whole TCGA patients is 0.689 and 0.733 , respectively. The expression 
of 9 significant IncRNA, the count of somatic mutations and the level of UBQLN4 were also plotted in the Fig. 4B,D.

\section{The prognostic value of the GILncSig compared with other model}

Two published articles about the model of IncRNA signatures with PC patients were compared with our model of GILncSig. One article come from Zhou's study including three IncRNA (MIR600HG, AL137789.1 and AC079015.1) ${ }^{[12]}$, another was Song's study including five IncRNA(C9orf139, MIR600HG, RP5965G21.4, RP11-436K8.1, CTC-327F10.4) ${ }^{[13]}$. The results showed that the AUC of GILncSig was larger than the two other model.(Fig. 5).

\section{The value of GILncSig}

The cox regression analyses was used to screen for prognostic index of PC, the results of GILncSig and other clinical index(age, gender, stage and grade) was got as table3, only GILncSig was significantly factor in each set. Besides, the survival curve of low risk patients were better than high risk patients in different clinical group.(Fig. 6). Therefore the model of GILncSig have significant value for predicting the survival of PC patients.

\section{The value of The GILncSig with KRAS mutation status}

The proportion of TCGA patients with KRAS mutations in the low-risk group(67\%) was significantly lower than in the high-risk group $(83 \%)(p=0.022)$. In the training group, the $p$ value is $p=0.435$ (low risk group:76\% vs high-risk group:85\%); in the testing group, the $\mathrm{p}$ value is 0.027 (low risk group:56\% vs high risk group:81\%).(Fig. 7A,B,C). So the GILncSig model was correlated with the KRAS mutations of PC, KRAS mutation was an important factor in the diagnosis, prognosis and treatment of pancreatic cancer $^{[14,15]}$, than the survival curves were drawn according the the GILncSig and KRAS, the prognosis in the KRAS mutation/GU-like group was poorest, and the prognosis in the KRAS wild/GS-like was best.

\section{Discussion}

Pancreatic cancer is a highly lethal disease with poor prognosis and high risk of metastasis, for which surgical resection is the only hopeful treatment ${ }^{[3]}$. PC is insensitive to multiple non-surgical treatments, especially to radiotherapy. Chemotherapy, which offers vital improvement in prognosis of many kinds of carcinoma, is ineffective for PC due to lack of effective target. The early diagnosis of PC is generally poor conducted due to the absence of significantly biomarkers. Therefore, it is urgent to explore the molecular mechanism in order to understand the pathogenesis and prognosis of PC. LncRNA has been known that serve important roles in diverse biological processes of human body, and dysregulated IncRNA expression may contribute to the development of numerous diseases especially malignant tumor ${ }^{[16]}$. Existing reports suggests that genome instability is a reliable predictor for predicting tumor prognosis and recurrence ${ }^{[17]}$, but there is no accurate quantitative standard to measure the extent of variation so far. Researchers have found that IncRNA is related to the expression of DNA damage-related genes and 
protein, which can directly cause genome instability ${ }^{[18]}$. Our study comprehensively analyzed the IncRNA expression profile and somatic mutations of patients with PC, aiming to find the IncRNA related to the tumor development and provide accurate treatment targets and prognostic factors.

178 samples were obtained from the TCGA database. The GU-like group and the GS-like group included 45 and 46 patients respectively according to the number of somatic mutations. As an important indicator of genome instability, UBQLN4 expression was significantly higher in GU-like groups, which proved the relationship between somatic mutation number and genome instability strongly ${ }^{[19]}$. After functional analysis of the mRNA co-expressed with differentially expressed IncRNAs, 10 mRNAs with the highest correlation were screened out to built the expression network. It can be found that these genes are mainly enriched in a variety of cell signal transduction and metabolism through KEEG analysis. A few studies have shown that some hormones, especially insulin, play an important role in the growth, metastasis and treatment resistance of $\mathrm{PC}^{[20]}$. Insulin /IGF pathway has become a key therapeutic target for pancreatic cancer. Abnormal endocrinology will lead to genome instability eventually.

9 IncRNA were identified to be associated with patient and GILncSig was established based on Cox proportional hazard regression analysis. The high-risk and low-risk groups were divided according to the median GILncSig risk score, which showed significant survival differences not only in the training set, but also in the validation set and the entire TCGA database. The expression of UBQLN4 and the number of somatic mutations were also significantly different between different groups, indicating that the use of GILncSig for risk assessment has good reliability and accuracy. Our model of GILncSig was compared with two other model of IncRNA signatures from Zhou's and Song's articles respectively. Zhou's model included three IncRNA, while Song's model summarized five. The AUC of our model is much larger than that of the other two models, indicating that our model has better prognostic assessment ability after incorporating more IncRNA related to gene instability.

KRAS gene plays an important role in many cancers including pancreatic cancer, gastric cancer and colorectal cancer ${ }^{[21]}$. Patients with KRAS mutation-type are generally less sensitive to subsequent treatments such as chemotherapy and have a worse overall survival than patients with KRAS wildtype ${ }^{[22]}$. Regardless of the training set, testing set, or all TCGA patients, KRAS gene mutations were significantly higher in the GU-like group than in the GS-like group according to the somatic mutation number. Furthermore, we observed that the KRAS wild-type patients of GS-like group had the best prognosis. However, at the same time, there was little difference in overall survival between patients with KRAS wild-type and KRAS mutation-type in the GU-like group, reflecting that compared with the widely recognized indicator-KRAS, assessment of genome instability might be more critical to prognosis. There are also some limitations in our study. The results obtained were only verified in the testing set and TCGA database, lacking verification of more external data sets, especially relevant data collected from clinical work. At the same time, no experiments were carried out to further clarify the mechanism of IncRNA and genome instability. Therefore, further studies on IncRNA associated with gene instability in pancreatic cancer are required in the future. 


\section{Conclusion}

In conclusion, our GILncSig included 9 IncRNA were found related with the survival and genome instability of PC(SOCS2-AS1, AC127024.6, TRPC7-AS1, AL359504.1, AL117382.2, LYPLAL1-AS1, LINC02014, CASC8, KRT7-AS), and there are significant differences in prognosis and clinical features between high-risk and low-risk groups differentiated by median risk score. This study can help assess the prognosis and treatment of patients with PC.

\section{Declarations}

\section{Ethics statement}

Our study was reviewed and approved by the Ethics Committee of the First Affiliated Hospital of Anhui Medical University. The data involved in the research were all available on the Internet.

\section{Consent of publication}

Written informed consent was obtained from the authors for the publication of any potentially indentifiable images or data included in our manuscript.

\section{Data availability statement}

Publicly available datasets were analyzed in this study. This data can be found in The Cancer Genome Atlas (TCGA) database (https://portal.gdc.cancer.gov/).

\section{Competing interests}

The authors declare that the research was conducted in the absence of any commercial or financial relationships that could be construed as a potential conflict of interest.

\section{Funding}

Natural Science Foundation of Anhui Province, No. 2108085QH337

\section{Acknowledgements}

Not applicable

\section{References}


1. Giannuzzo A, Pedersen S F, Novak I. The P2X7 receptor regulates cell survival, migration and invasion of pancreatic ductal adenocarcinoma cells[J]. Mol Cancer, 2015, 14: 203.

2. Dodson L F, Hawkins W G, Goedegebuure P. Potential targets for pancreatic cancer immunotherapeutics[J]. Immunotherapy, 2011, 3(4): 517-37.

3. Mcguigan A, Kelly P, Turkington R C, et al. Pancreatic cancer: A review of clinical diagnosis, epidemiology, treatment and outcomes[J]. World J Gastroenterol, 2018, 24(43): 4846-4861.

4. Azizian A, Rühlmann F, Krause T, et al. CA19-9 for detecting recurrence of pancreatic cancer[J]. Sci Rep, 2020, 10(1): 1332.

5. Goodall G J, Wickramasinghe V O. RNA in cancer[J]. Nat Rev Cancer, 2021, 21(1): 22-36.

6. Negrini S, Gorgoulis V G, Halazonetis T D. Genomic instability-an evolving hallmark of cancer[J]. Nat Rev Mol Cell Biol, 2010, 11(3): 220-8.

7. Ottini L, Falchetti M, Lupi R, et al. Patterns of genomic instability in gastric cancer: clinical implications and perspectives[J]. Ann Oncol, 2006, 17 Suppl 7: vii97-102.

8. Wang $T$, Wang $G$, Zhang $X$, et al. The expression of miRNAs is associated with tumour genome instability and predicts the outcome of ovarian cancer patients treated with platinum agents[J]. Sci Rep, 2017, 7(1): 14736.

9. Schmitt A M, Chang H Y. Long Noncoding RNAs in Cancer Pathways[J]. Cancer Cell, 2016, 29(4): 452463.

10. Betts J A, Moradi Marjaneh M, Al-Ejeh F, et al. Long Noncoding RNAs CUPID1 and CUPID2 Mediate Breast Cancer Risk at 11q13 by Modulating the Response to DNA Damage[J]. Am J Hum Genet, 2017, 101(2): 255-266.

11. Sharma V, Khurana S, Kubben N, et al. A BRCA1-interacting IncRNA regulates homologous recombination[J]. EMBO Rep, 2015, 16(11): 1520-34.

12. Zhou C, Wang S, Zhou Q, et al. A Long Non-coding RNA Signature to Improve Prognostic Prediction of Pancreatic Ductal Adenocarcinoma[J]. Front Oncol, 2019, 9: 1160.

13. Song J, Xu Q, Zhang $\mathrm{H}$, et al. Five key IncRNAs considered as prognostic targets for predicting pancreatic ductal adenocarcinoma[J]. J Cell Biochem, 2018, 119(6): 4559-4569.

14. Matsumoto K, Kato H, Nouso K, et al. Evaluation of Local Recurrence of Pancreatic Cancer by KRAS Mutation Analysis Using Washes from Endoscopic Ultrasound-Guided Fine-Needle Aspiration[J]. Dig Dis Sci, 2020, 65(10): 2907-2913.

15. Buscail $L$, Bournet $B$, Cordelier P. Role of oncogenic KRAS in the diagnosis, prognosis and treatment of pancreatic cancer[J]. Nat Rev Gastroenterol Hepatol, 2020, 17(3): 153-168.

16. Bhan A, Soleimani M, Mandal S S. Long Noncoding RNA and Cancer: A New Paradigm[J]. Cancer Res, 2017, 77(15): 3965-3981.

17. Bakhoum S F, Cantley L C. The Multifaceted Role of Chromosomal Instability in Cancer and Its Microenvironment[J]. Cell, 2018, 174(6): 1347-1360. 
18. Zhou Z, Liu H, Wang C, et al. Long non-coding RNAs as novel expression signatures modulate DNA damage and repair in cadmium toxicology[J]. Sci Rep, 2015, 5: 15293.

19. Li J, Tong Y, Wang Z, et al. A Comprehensive Multiomics Analysis Identified Ubiquilin 4 as a Promising Prognostic Biomarker of Immune-Related Therapy in Pan-Cancer[J]. J Oncol, 2021, 2021: 7404927.

20. Mutgan A C, Besikcioglu H E, Wang S, et al. Insulin/IGF-driven cancer cell-stroma crosstalk as a novel therapeutic target in pancreatic cancer[J]. Mol Cancer, 2018, 17(1): 66.

21. Timar J, Kashofer K. Molecular epidemiology and diagnostics of KRAS mutations in human cancer[J]. Cancer Metastasis Rev, 2020, 39(4): 1029-1038.

22. Binefa G, Rodríguez-Moranta F, Teule A, et al. Colorectal cancer: from prevention to personalized medicine[J]. World J Gastroenterol, 2014, 20(22): 6786-808.

\section{Tables}

Tables are only available as a download in the Supplemental Files section.

\section{Figures}




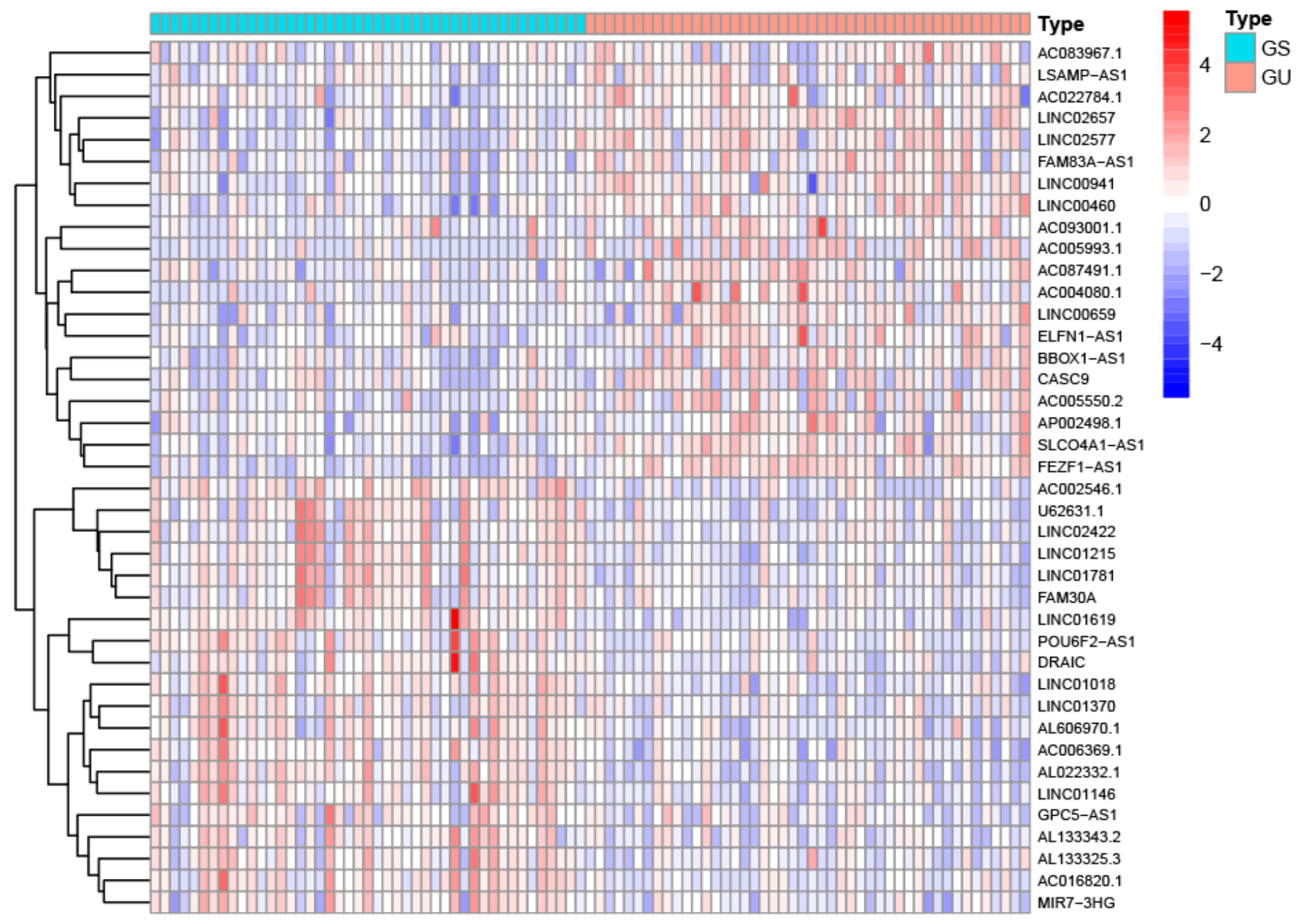

Figure 1

The top 20 of upregulated and downregulated IncRNAs between the GU-like group and GS-like group.

\section{Figure 2}

Identification and functional annotations of genome instability-related IncRNAs in patients with pancreatict cancer. (A): Hierarchical clustering analysis of 178 pancreatic cancer patients according to the different IncRNAs. (B): Boxplots of somatic mutations in the GU-like group and GS-like group. Somatic cumulative mutations in the GU-like group are significantly higher than those in the GS-like group. (C): Boxplots of UBQLN4 expression level in the GU-like group and GS-like group. The expression level of UBQLN4 in the GU-like group is significantly higher than that in the GS-like group. (D): Co-expression network of genome instability-related IncRNAs and mRNAs based on the Pearson correlation coefficient. The red circles represent mRNAs, and the blue circles represent IncRNAs. (E): Functional enrichment analysis of GO and KEGG for mRNAs co-expressed IncRNAs. 

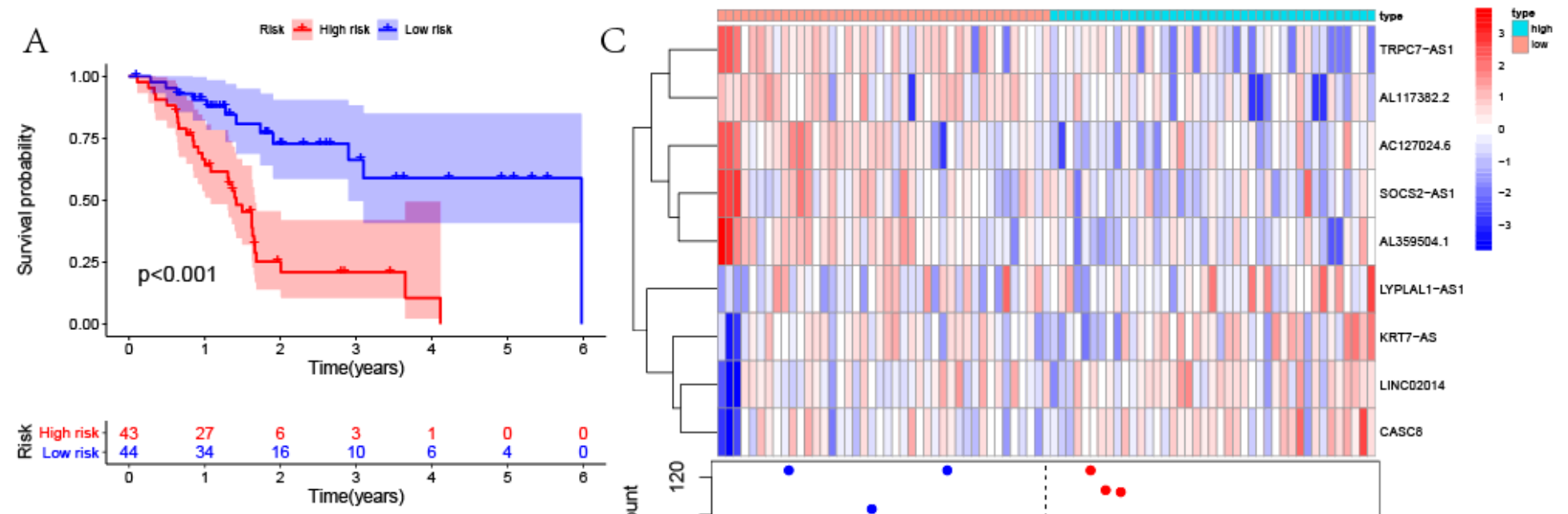

$\mathrm{B}$

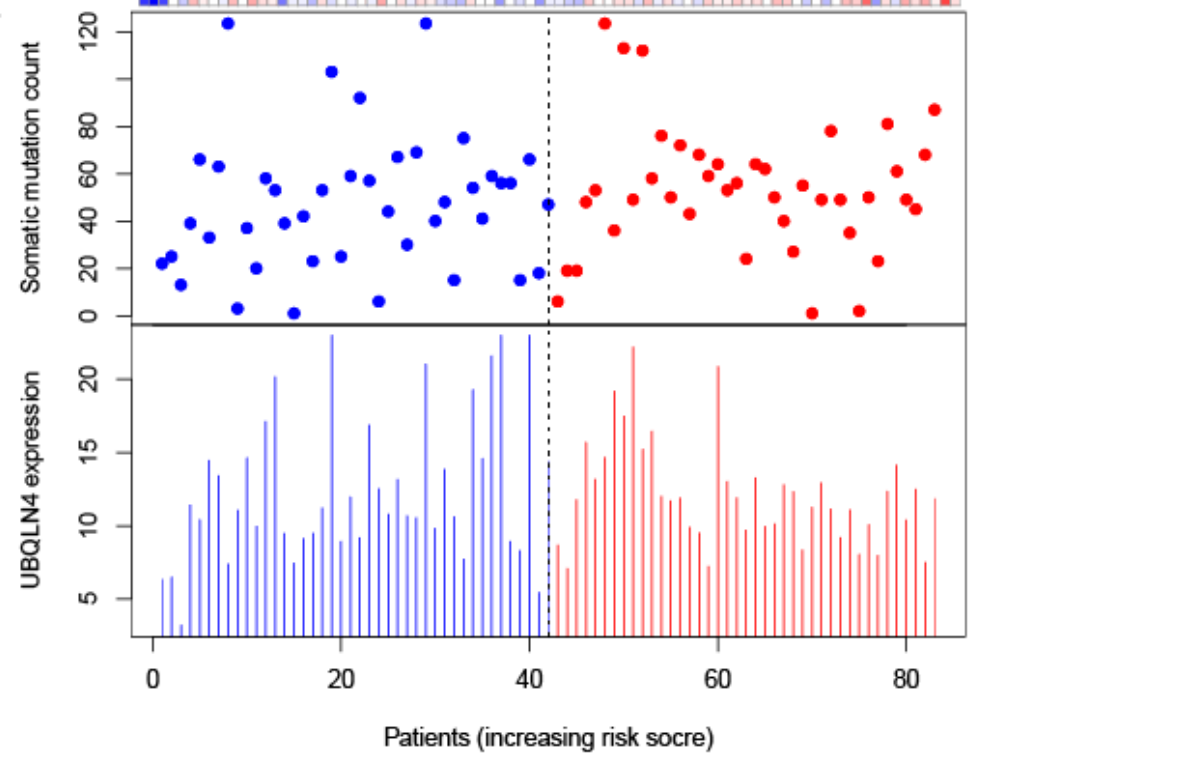

\section{Figure 3}

Identification of the genome instability-derived IncRNA signature (GILncSig) for outcome prediction in the training set. (A): Kaplan-Meier survival analysis of patients with low or high risk predicted by the GILncSig in the training set. (B): Time-dependent ROC curves analysis of the GILncSig. (C): LncRNA expression patterns and the distribution of somatic mutation and UBQLN4 expression with increasing GILncSig score. 
A

Risk \pm High risk \pm Low risk

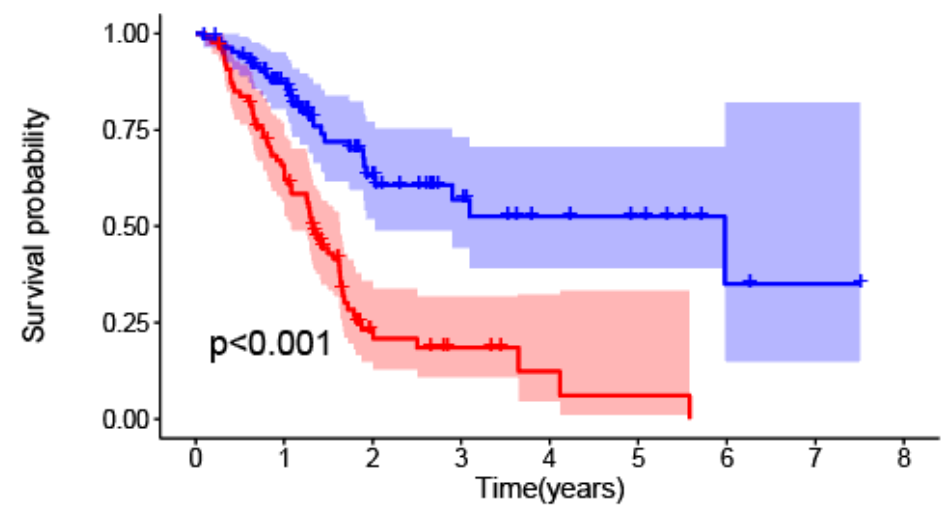

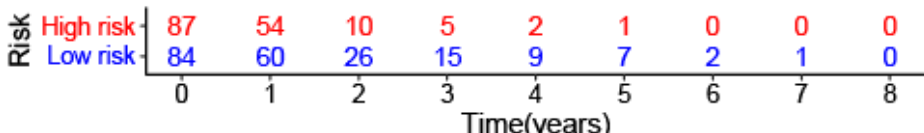

B

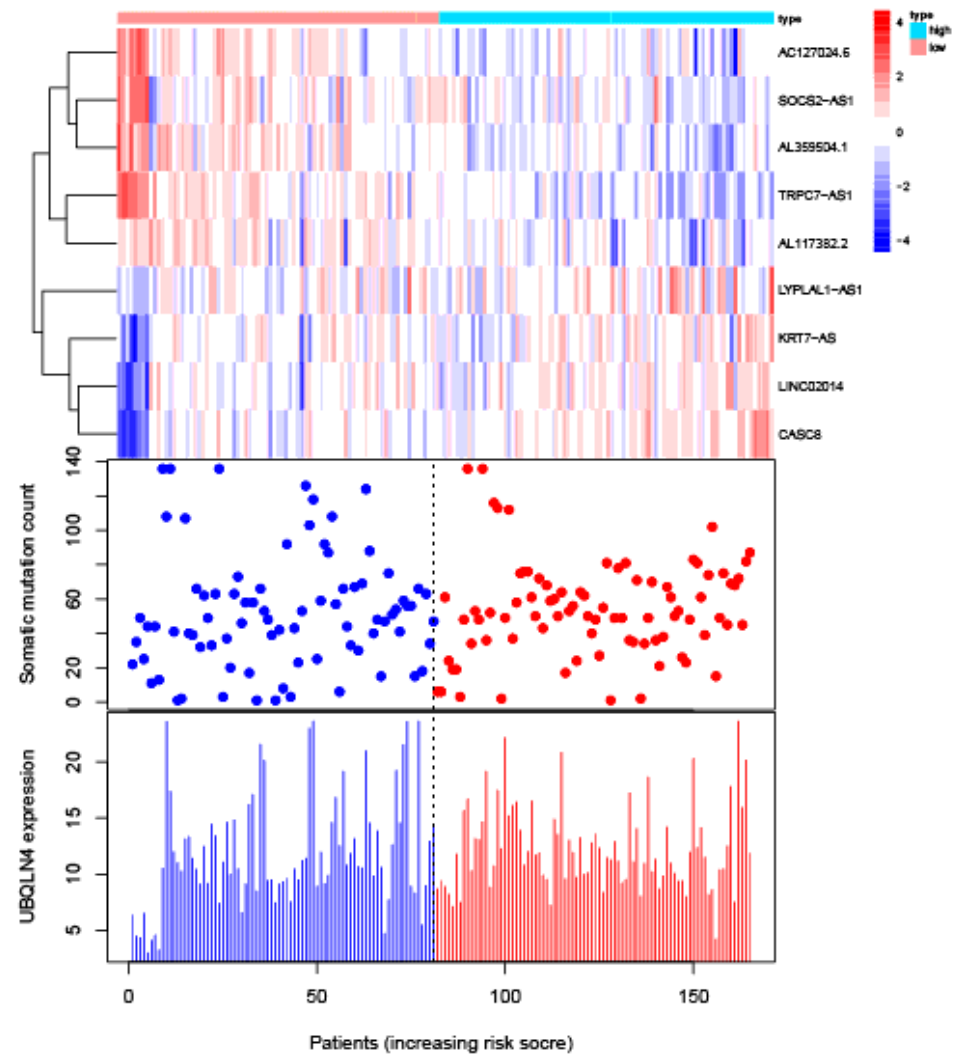

C

Risk \pm High risk \pm Low risk

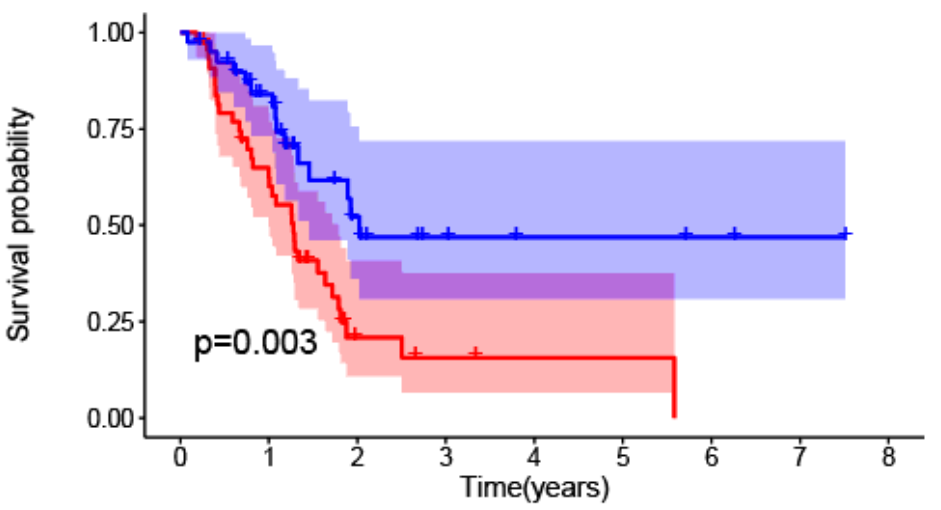

$\mathrm{D}$

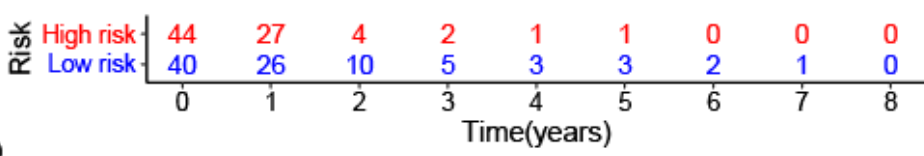

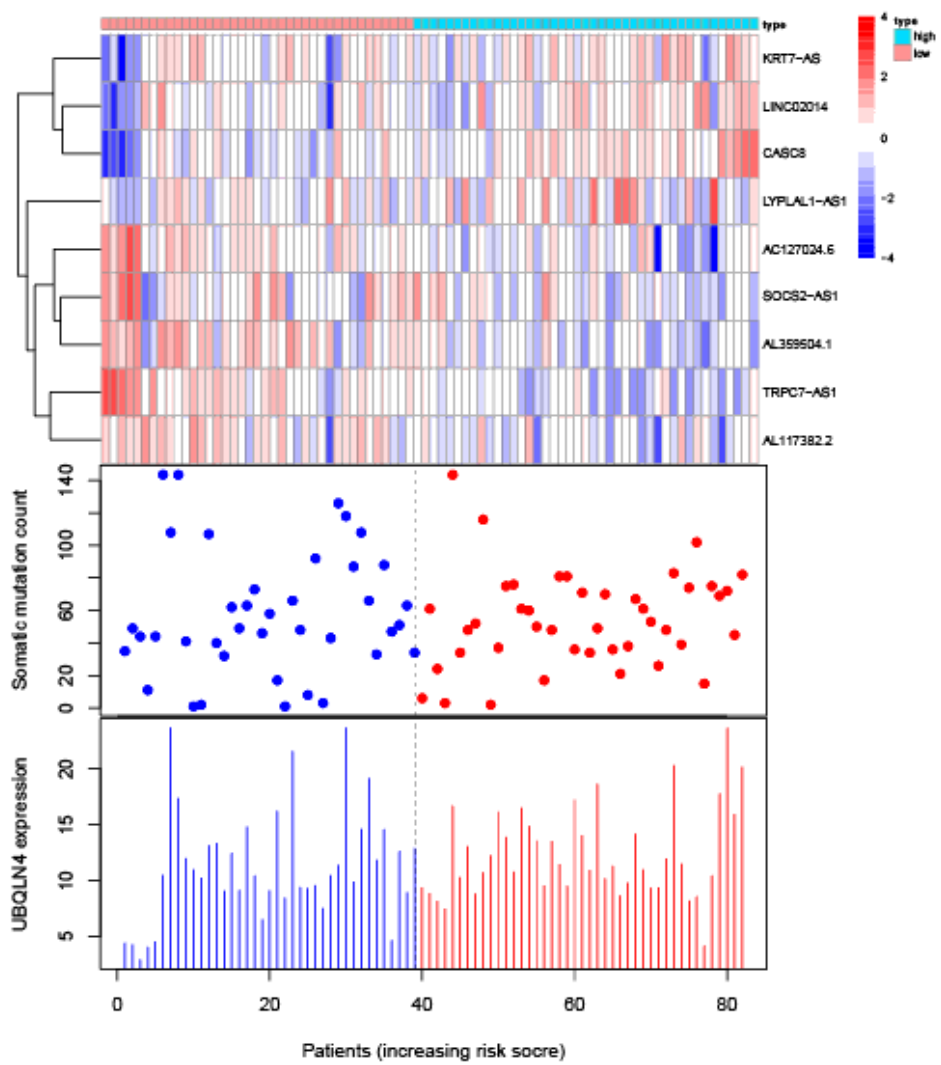

Figure 4

Kaplan-Meier estimates of overall survival of patients with low or high risk predicted by the GILncSig in the testing set $(A)$ and TCGA set $(C)$. LncRNA expression patterns and the

distribution of somatic mutation count distribution and UBQLN4 expression for patients in high- and lowrisk groups in the testing set (B) and TCGA set (D). 


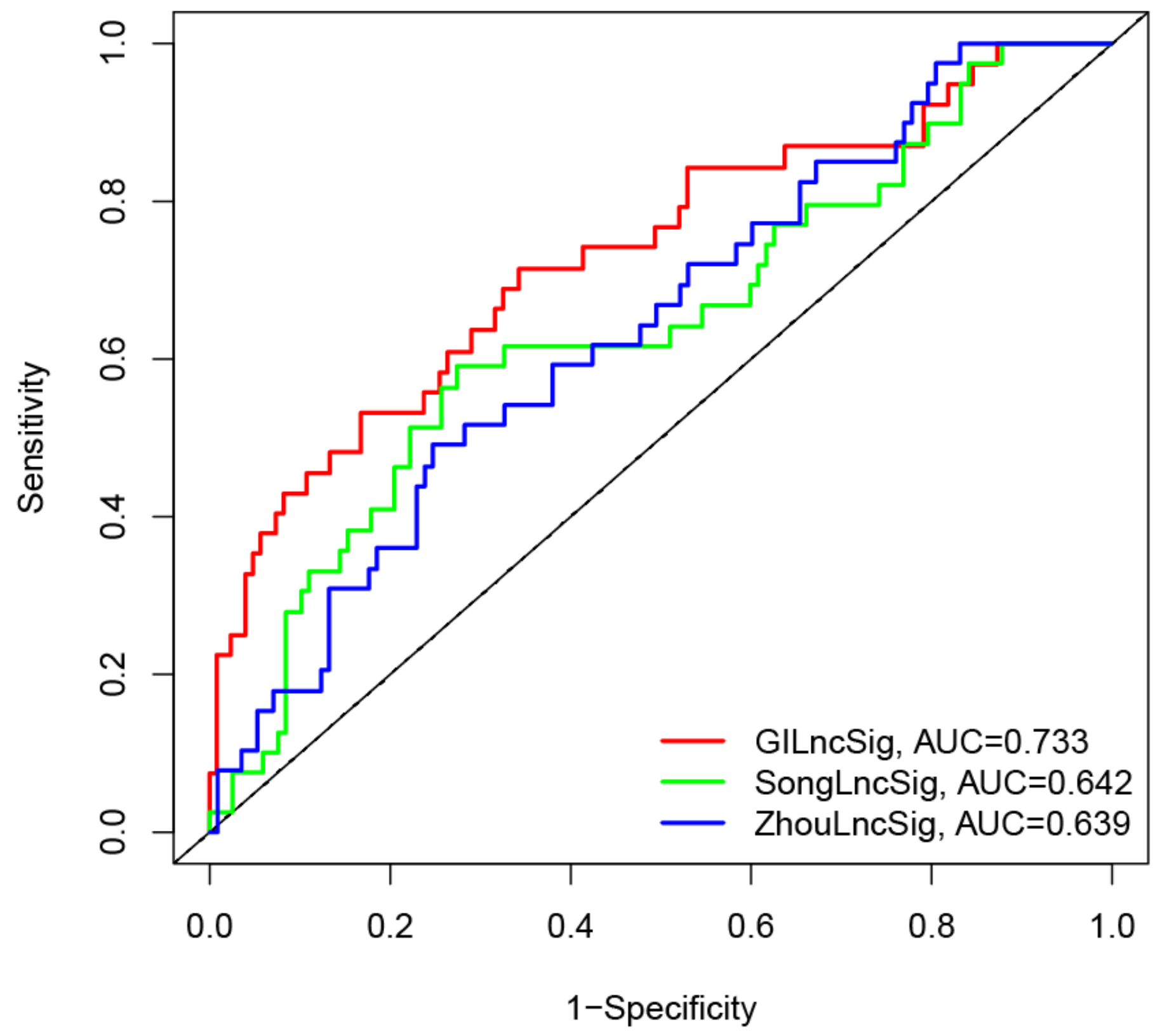

Figure 5

The ROC analysis of overall survival for the GILncSig, SongLncSig and ZhouLncSig. 
Patients with FEMALE

Risk - high - low

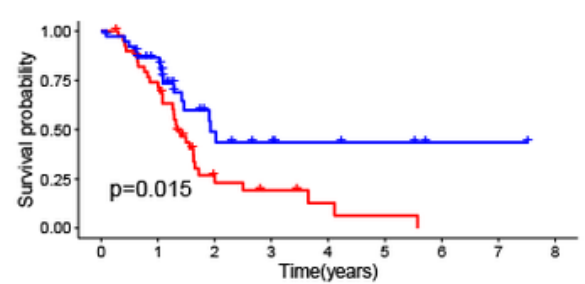

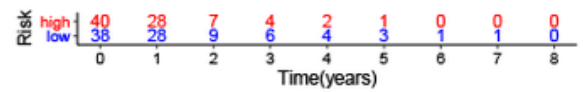

Patients with T1-2

Risk + high + low

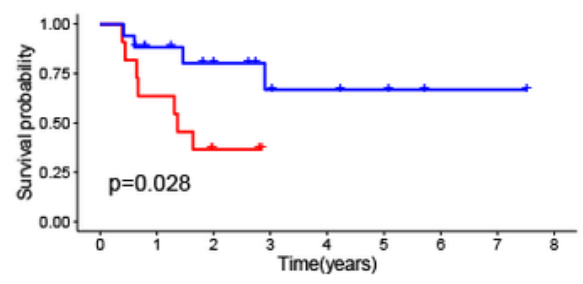

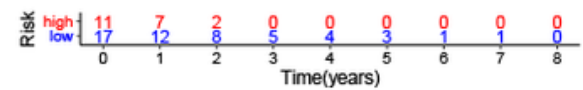

Patients with NO

Risk - high - low
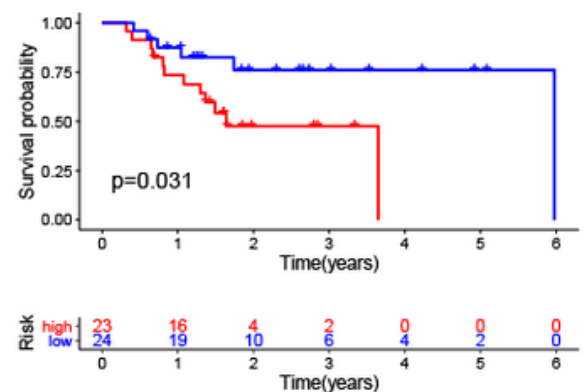

Patients with G1-2

Risk + high + low

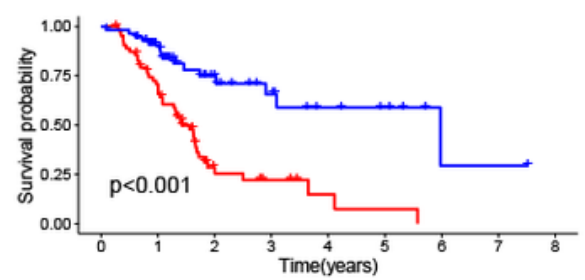

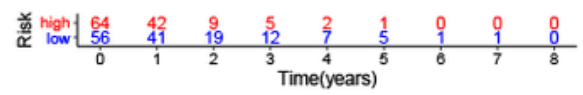

Patients with MALE

Risk - high - low
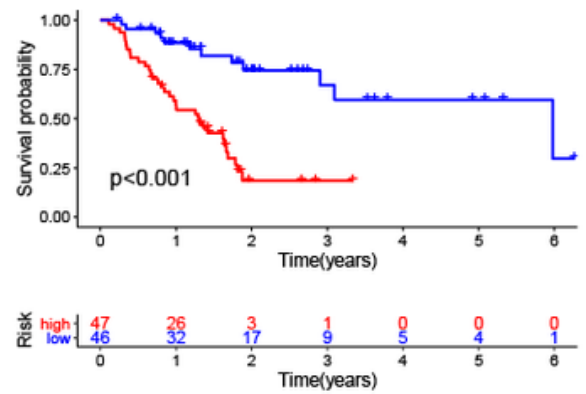

Patients with $\mathrm{T} 3-4$

Risk + high + low
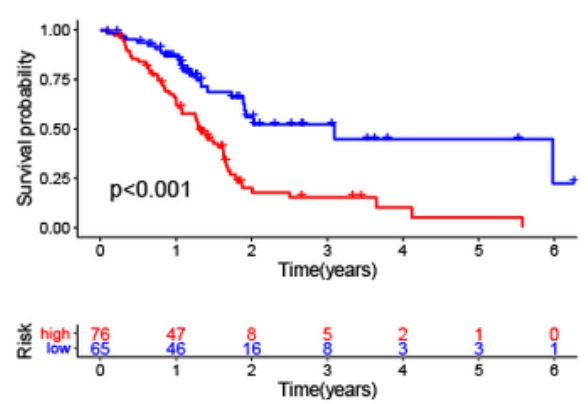

Patients with $\mathrm{N} 1$

Risk - high - low

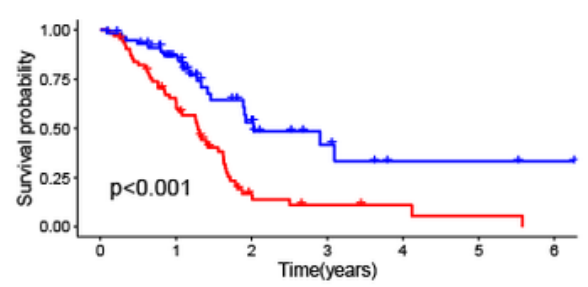

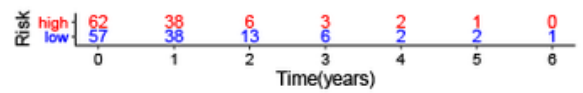

Patients with G3-4

Risk + high + low

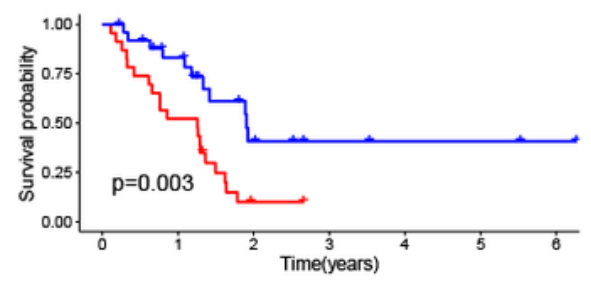

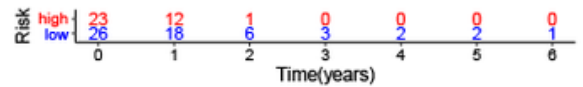

\section{Figure 6}

Kaplan-Meier survival analysis of overall survival in high- and low-risk groups for patients in different clinical group. 
A

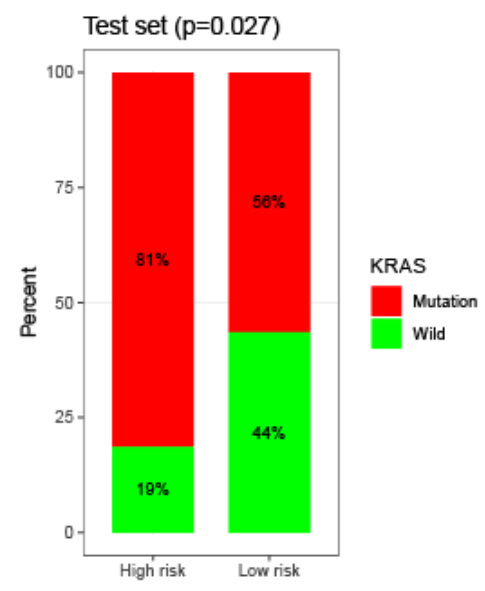

B

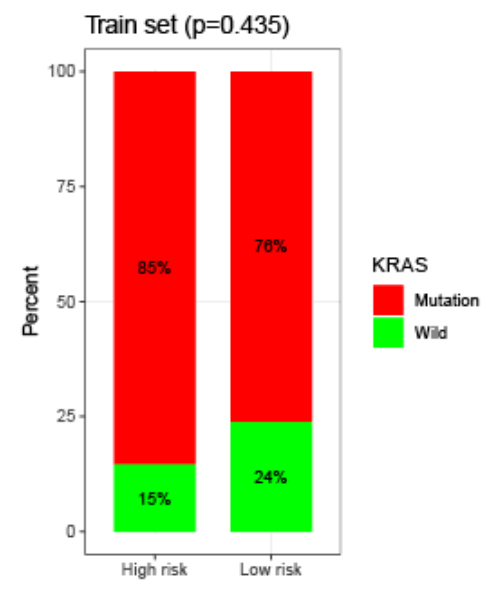

C

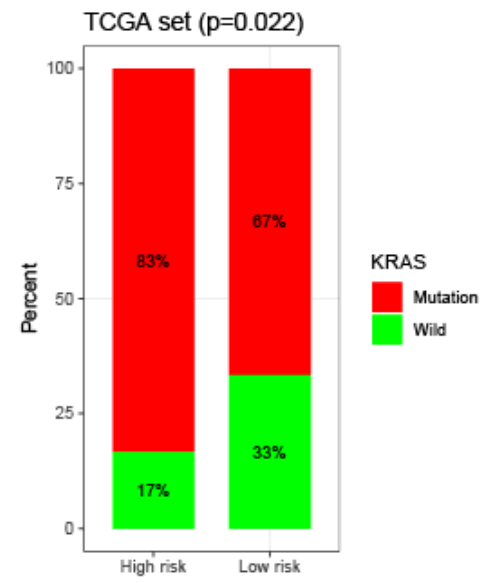

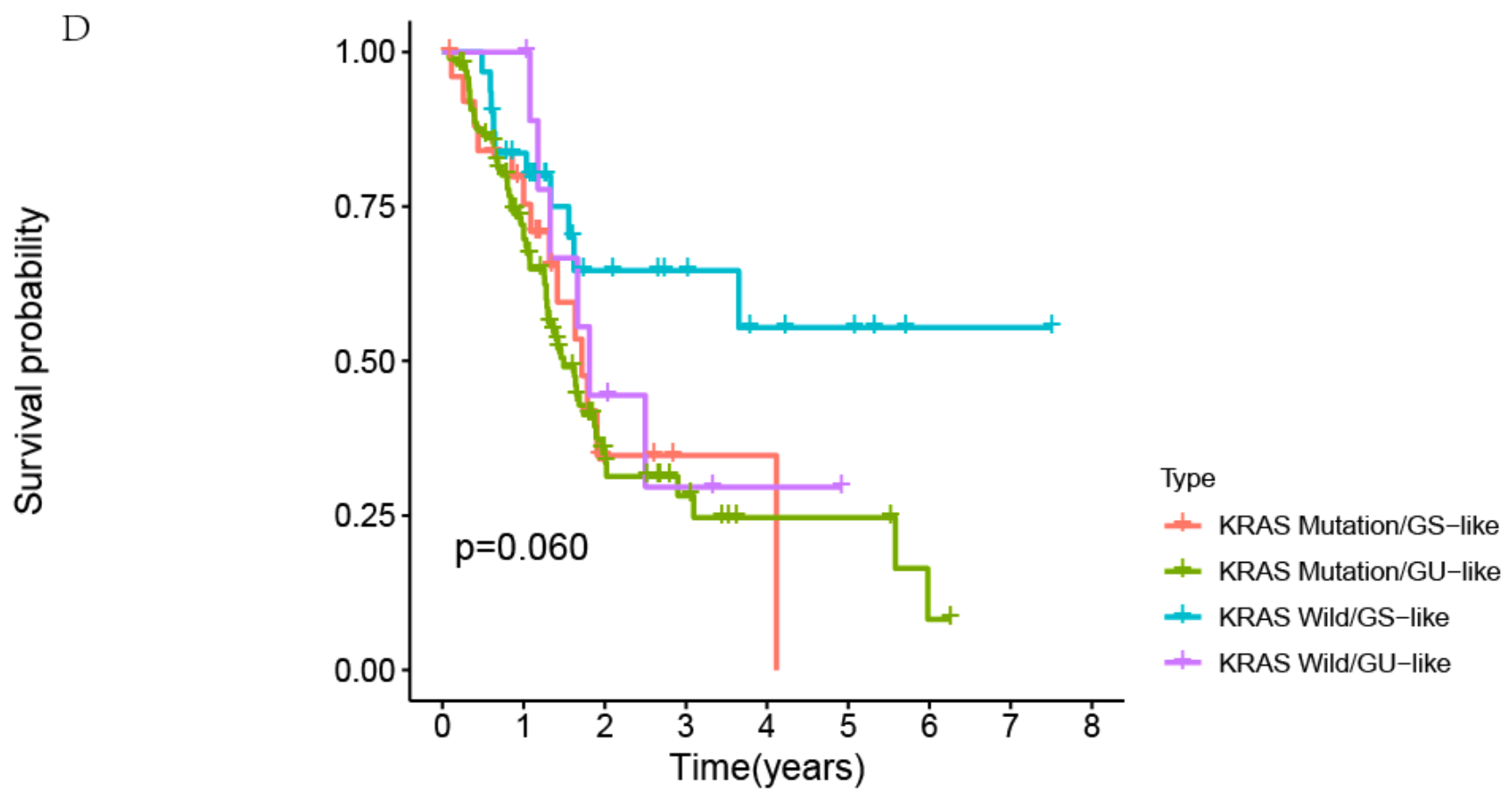

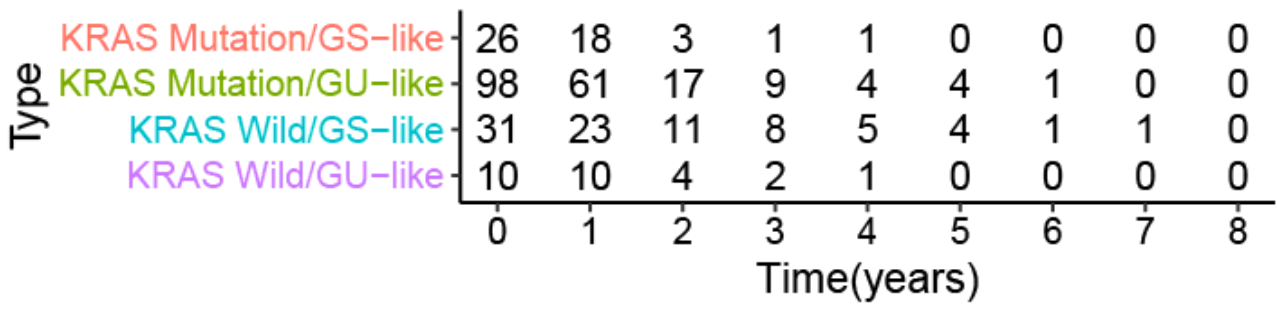

Figure 7

(A): The proportion of KRAS mutation in high- and low-risk groups in the testing set. (B): The proportion of KRAS mutation in high- and low-risk groups in the training set. (C): The proportion of KRAS mutation in high- and low-risk groups in the TCGA set. (D): Kaplan-Meier curve analysis of overall survival is shown for patients classified according to KRAS mutation status and the genome instability. 


\section{Supplementary Files}

This is a list of supplementary files associated with this preprint. Click to download.

- Table1.xls

- Table2.xlsx

- Table3.xlsx 